\title{
UNA MIRADA ALTERNATIVA ACERCA DEL RESIDUO COGNITIVO CUANDO SE INTRODUCEN NUEVAS TECNOLOGÍAS. EL CASO DE LA RE- SOLUCIÓN DE PROBLEMAS EN CIENCIAS.
}

Resumen: En el presente texto, se presentan vínculos entre elementos provenientes de referenciales psicológicos en la necesidad de contar con una mejor teoría que de cuenta de la relación entre los residuos cognitivos, la influencia de las tecnologías de la información y la comunicación y la enseñanza que se propone en relación con la educación en general, y con la Enseñanza de las Ciencias y la investigación en resolución de problemas en particular. Se parte del conocimiento de "la persona y sus capacidades” y nos dirigimos hacia el conocimiento de "la persona-cuando-utiliza-esa-herramienta (computadora)”. En ese recorrido se vislumbran la incorporación de la novedad a través de la problematización y la construcción de criterios propios surgidos en actividad grupal como iluminando decisiones didácticas en esa dirección.

Palabras clave: Efecto cognitivo, nuevas tecnologías, representaciones mentales, resolución de problemas en ciencias.
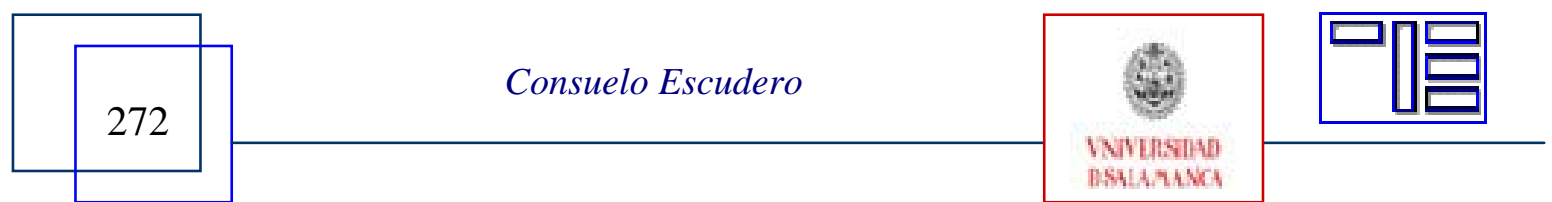


\section{A ALTERNATIVE GLANCE ABOUT OF THE COGNITIVE RESIDUE WHEN NEWS TECHNOLOGIES ARE INTRODUCED. THE CASO OF THE SCIENCES PROBLEM SOLVING.}

Abstract: In this paper are introduced links between elements coming from psychological referential due to the need of counting with a better theory to account for the relation among cognitive residues, CIT's influence and the teaching proposed in relation with the education in general and with the teaching of Sciences and the research in solving problems in particular. The departure is, in this case, from the knowledge of the "person and his/her capacities" and the research is towards the knowledge of the "person-whenshe/he uses-the tool (computer)". It is in that context, that the incorporation of the novelty through the problem solving method and the construction of own criteria aroused from grouped activity is viewed as lighting didactic decisions in that direction.

Keywords: Cognitive effect, news technologies, mental representations, problem solving in sciences.

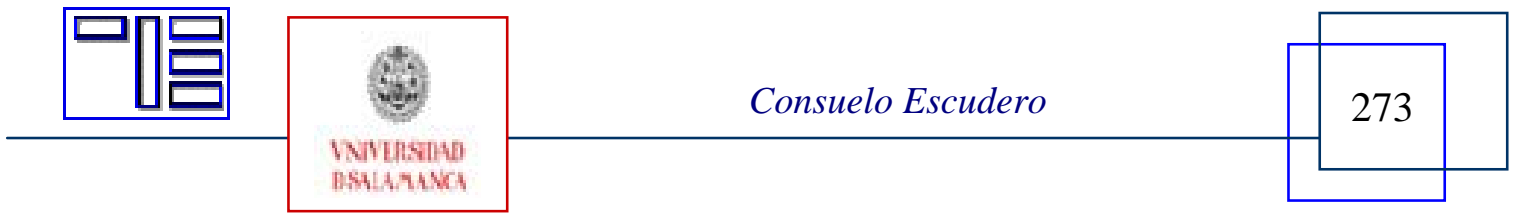




\section{UN REGARD ALTERNATIVE SUR LE RÉSIDU COGNITIVE QUAND ON IN- TRODUI NOUEVELLES TECHNOLOGIES. LE FAIT DE LA RESOLUTIONS DES PROBLÈMES DANS LES SCIENCIES.}

Sommaire : Dans le présent texte on présente des liens entre des éléments qui on apporté par des références psichologiques nécessaires pour avoir une meilleur théorie que exprime des relations entre les résidus cognitives, l' influence de les TIC's et l' enseignement qui on propose en relation avec l'education en générale et avec l' enseignement de les sciences et l'investigation en resolution de des problèmes en particulier. On part de la connaissance de «la personne et ses capacités» et nous dirigions par la connaissance de «la personne-quand-utilise-cette-outil» (de l'ordinateur). Dans cette parcours on anticipe l'incorporation de la nouveauté par l'intermédiaire de la question problématique et la construction de des critères propres et qui ils sont produits à l'activité en équipe et qu' ils iluminent des décisions didactiques en cette direction.

Mots clefs: Effet cognitive, nouevelles technologies, representations mentals, resolutions des problèmes en sciencies.
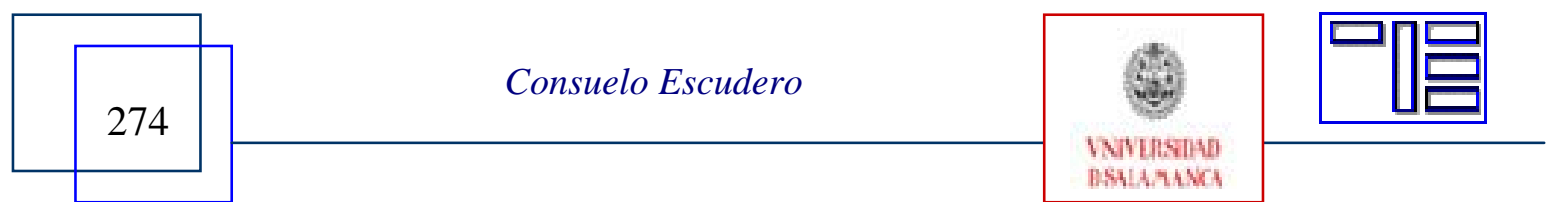


\section{UNA MIRADA ALTERNATIVA ACERCA DEL RESIDUO COGNITIVO CUANDO SE INTRODUCEN NUEVAS TECNOLOGÍAS. EL CASO DE LA RE- SOLUCIÓN DE PROBLEMAS EN CIENCIAS.}

Consuelo Escudero

cescude@unsj.edu.ar

Universidad Nacional de San Juan (UNSJ) - Argentina.

\section{1.- INTRODUCCIÓN.}

Las dos últimas décadas han quedado marcadas por la influencia que la tecnología ha tenido en todos los espacios de la actividad humana.

La modificación de los procesos de enseñanza y de aprendizaje en relación con la educación en general, y con la Enseñanza en Ciencias y la investigación en resolución de problemas en particular; aparece (o se manifiesta) como una cuestión relevante. Tener en cuenta que los avances tecnológicos nos están demandando una nueva perspectiva que los contemple no solo como herramientas didácticas, sino como recurso productor de conocimientos en tanto contribuye enormemente a la explicitación de aquellas "relaciones y estructuras" que avizoraba la escuela pitagórica. Tanto es así, que Rabardel (2002) cita el Kittle House Manifesto, en el que se llama a una refundación de la psicología en este terreno.

Admitimos fácilmente que las TIC pueden mejorar la formación de los alumnos. Hay estudios (por ejemplo Epper y Bates, 2004, Sierra, 2004, López et al. 2006, Buzzo, 2007) que señalan que el uso de computadoras mejora el aprendizaje de los estudiantes. Pero nos cuesta aceptar que una utilización comprometida con la creatividad requiere una dedicación tanto o más cuidada que aquella, sobre todo, para imaginar con tecnologías, para diseñar, para resolver problemas.

...el ordenador es un meta-medio simbólico, en tanto que actúa como herramienta cognitiva que trasciende las limitaciones del procesamiento humano (memoria, carga atencional, fatiga) al tratar la información, es decir, al recibir, almacenar, transformar y generar datos, mediante la manipulación de símbolos que son decodificados en otros símbolos comprensibles para el usuario (enactivos, lingüísticos, matemáticos e icónicos) (Sierra, 2000).

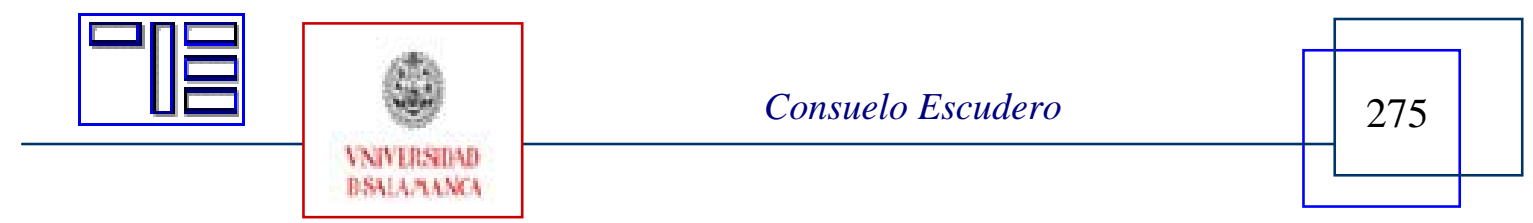


Esta situación nos compromete a intentar una mirada desde otra perspectiva, que permita introducirnos en el universo de las relaciones entre los conceptos implicados, específicamente, en la resolución de problemas y las nuevas tecnologías de la información y la comunicación. Sin dejar de tener en cuenta que para que se produzca este encuentro, quien se enfrenta a las situaciones problemáticas debe tener al menos un mínimo interés por resolverlas (Pozo y Gómez Crespo, 1998; Perales, 2000; Ballesteros, 2003).

Teniendo en cuenta este escenario surgen preguntas que comienzan a orientar nuestra búsqueda:

- ¿ ¿Qué diferencias marcan la utilización directa de la computadora y la realización de un trabajo primero en borrador (lápiz y papel)?

- ¿ ¿Qué variables introduce la utilización de la computadora para la realización de algunas producciones?

- ¿ ¿Existían esas variables previamente a dicha introducción?

- ¿Mejoran las producciones o los mismos procesos de construcción del conocimiento cuando se utilizan tecnologías?

- ¿Cambian los procesos de resolución de problemas en el área de las Ciencias Básicas diferenciando entre dos métodos de resolución: a través de software educativo (simulaciones) y en forma tradicional (lápiz y papel)?

- ¿ ¿Qué sucede cuando son las funciones de un software las que orientan el proceso de la resolución de problemas?

- ¿QQué actividades de simulación pueden contribuir eficazmente en el proceso de enseñanza-aprendizaje proporcionado un aprendizaje más significativo?

- ¿ ¿Cómo y cuánto contribuyen?

- ¿Qué características deberían reunir las actividades de simulación y modelado para facilitar la adquisición de conocimiento científico?

- ¿Qué hay de las economías cognitivas que aparecen casi como una "ideología” que nuestros "sujetos de aprendizaje" hoy defienden: el esfuerzo frente a la eficiencia, lo molecular frente a lo molar, las imágenes frente a las palabras?

En síntesis, ¿Qué cambia o qué no cambia en nuestras mentes en los intercambios con tecnologías?

A menudo se sigue la vía que consiste en reorganizar las tareas con el objetivo que los resultados no dependan del operador, y donde la potencia contemporánea de la maquinaria permite cada

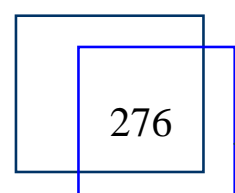

Consuelo Escudero

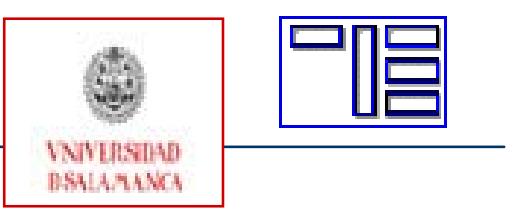




\begin{tabular}{|c|c|c|}
\hline 口包 & $\begin{array}{l}\text { Revista Electrónic } \\
\text { Educación y Cultura en }\end{array}$ & $\begin{array}{l}\text { ducación. } \\
\text { la Información. }\end{array}$ \\
\hline & http://www.usal.es/teoriaeducacion & Vol. 10. No 1. Marzo 2009 \\
\hline
\end{tabular}

vez más imaginarla como una vía real. Esta reside (se asienta o radica) en mirar la actividad humana como un residuo (Clot, 1992).

Al final, no deja a los operadores más que el conjunto heteróclita de las tareas demasiado complejas, imposibles de automatizar, nos recuerda Bainbrige (1982).

Desde los enfoques tecnocéntricos de los objetos y sistemas el hombre ocupa un lugar residual, y su actividad real no tiene status propio. En términos de la técnica, la actividad pierde su identidad: el hombre es pensado en referencia a las cosas en los términos de las propias cosas. En los enfoques antropocéntricos la situación no es muy diferente, aunque las actividades de diseño aparezcan bastante bien analizadas, todavía la esfera del uso queda poco explorada y mal comprendida; son desconocidos los procesos de producción de conocimiento y, su existencia -en ciertos casos- negada.

Los interrogantes anteriores, creemos, pueden ser de sumo potencial para pensar propuestas didácticas y sus derivaciones cognitivas, cuando se introducen tecnologías, especialmente software, en el aula. La complejidad de su respuesta emana de su no encasillamiento en relaciones causales simples y de pulir las interpretaciones para que den cuenta de este residuo (o remanente[1]) enraizado en la cultura y no meramente en las paredes del aula.

Vamos a entender por residuo cognitivo un tipo de efecto vinculado con el intercambio con tecnologías que se caracteriza por la profundización en los compromisos mentales que el individuo asume en su modo de operar con ellas. No sólo de acceso y conectividad sino de transformación del valor de la información en conocimiento para la toma de decisiones, así como la posibilidad de incorporación de la novedad.

Uno de los núcleos más consistentes de la investigación en tecnología y educación ha sido el del impacto de la tecnología en la mente de los sujetos (Pea, 2001, Salomon, Perkins y Globerson1992, entre otros). La pregunta inicial que estos autores se plantean remite a si la tecnología puede hacernos cognitivamente más poderosos. La respuesta que sostienen es unívoca: no puede haber un solo mecanismo que dé cuenta de los efectos de la tecnología sobre la mente.

Salomon, Perkins y Globerson (1992), además, consideran la posibilidad de que la colaboración intelectual, sobre todo con las computadoras, deje residuos cognitivos entendidos como nuevas capacidades y habilidades transferibles a otras situaciones. Cobra así fuerza la idea -de fondo- de que el conocimiento está distribuido, circunscribiendo mejor los objetos con mente, así como que la cognición no es sólo un fenómeno individual. De esta forma, estaría dando cuenta en palabras de Pea (2001) de una verdadera distribución de la inteligencia que pone énfasis en el aspecto sistemático de la capacidad humana. Para lo cual admitiremos el remanente cognitivo como efecto posible del intercambio comprometido con tecnologías, especialmente informáticas. Parecería que un trabajo empírico y teórico considerable queda pendiente.

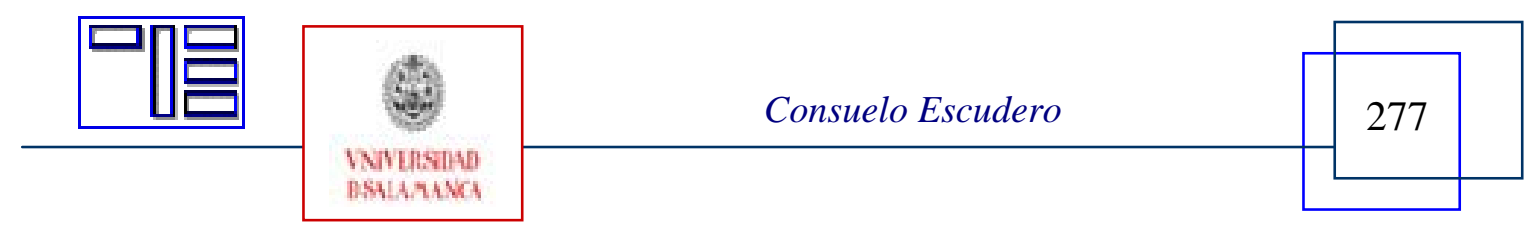


Nuestro propósito es seguir indagando acerca de algunos de los efectos cognitivos de enseñar y aprender con la tecnología; así como comprender mejor cómo el uso de determinadas aplicaciones mejora (o posibilita) nuevos tipos de acciones. Además de contribuir a fundamentar la construcción de una actual concepción de la resolución de problemas escolares en ciencias y su investigación a partir de nuevos marcos teóricos, principalmente psicológicos y sus implicancias pedagógicas y éticas. Respetando el escenario y el movimiento natural de las aulas, se parte del conocimiento de "la persona y sus capacidades" y nos dirigimos hacia el conocimiento de "la persona-cuando-utiliza-esaherramienta (computadora)”, expresado en términos de Rodriguez Illera (2004).

\section{2.- ESTADO ACTUAL DE LA INVESTIGACIÓN EN EL ÁREA.}

La resolución de problemas es una línea tradicional de investigación en Enseñanza de las Ciencias. Se trata de un tema muy debatido, no por eso menos actual y ya acabado. Tras un análisis restrospectivo de la literatura (Escudero, 2005 y Escudero y Moreira, 2004) observamos que se han multiplicado los aspectos investigados en la resolución de problemas, favoreciendo ampliamente el crecimiento del campo de investigación en ciencias y las expectativas en relación a la transferencia que se puede concretar en el aula.

A las cuestiones tradicionales, como el aprendizaje por modelos, la profundización en la comprensión de los conceptos, las propuestas que toman en cuenta la visión del investigador científico, los problemas abiertos, los problemas cualitativos, se han agregado estudios que incorporan las nuevas tecnologías o que se internan en la comprensión del texto del enunciado y algunos que han comenzado a integrar el aporte que proviene de trabajos sobre representaciones mentales de los estudiantes y también sobre el uso del lenguaje simbólico.

En relación a la incorporación de las nuevas tecnologías, enlazada fuertemente con el aprendizaje de los sistemas externos de representación como la notación numérica, la escritura, los diagramas usados (por ejemplo en Física); asoma como una cuestión interesante su abordaje desde el aula, a partir de las representaciones internas de los alumnos que comienzan a interactuar con los primeros conceptos en los distintos campos de cada disciplina.

Como en todo inicio, ya han surgido algunos trabajos de intervención, aunque no se puede aventurar la existencia de un cambio relevante en la incidencia que puedan llegar a tener estas acciones en el aprendizaje significativo. Ésta es una de las cuestiones a analizar, dado que el seguimiento de este tipo de procesos se va construyendo en la medida en que se van discriminando las alternativas que ofrecen las NTICs.

La función de las tecnologías de la información y comunicación en los procesos de enseñanza y de aprendizaje en muchas de sus modalidades de uso se restringe a la transmisión de información y comunicación. Mientras, hay modalidades en que las tecnolog-

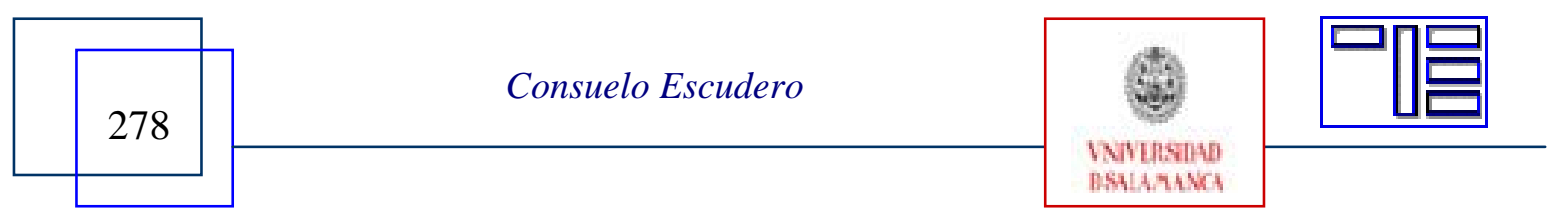


ías funcionan como herramientas que ayudan a pensar, con las cuales los estudiantes aprenden, en vez de aprender solamente las tecnologías en sí (Jonassen, 2000; Cataldi y Cabero, 2007).

Hay estudios (por ejemplo Bates, 2001; Sierra, 2004; Tello y Pérez, 2007) que -en rasgos generales- señalan que el uso de computadoras mejora el aprendizaje de los estudiantes, mientras otro grupo de autores (Jimoyannis y Komis, 2001, López et al 2006, Lion 2006), si bien, considera que el proceso de aprendizaje se modifica, se muestra más cauto a la hora de hacer apreciaciones. Se pregunta sobre cuándo se potencian las formas de aprender y de construir conocimiento, habiendo encontrado la idea de una economía cognitiva asociada a la posibilidad de verificación rápida de hipótesis. Existiría un tercer grupo más auspicioso que considera que puede ampliarse la capacidad cognitiva más allá de los límites viables sin el uso de recursos tecnológicos (por ejemplo Santos et al 2000; Araujo et al. 2007; Buzzo, 2007). En términos generales, puede ser aconsejable el uso de instrumentos que posibiliten visualizar aspectos de los fenómenos de la ciencia a los que difícilmente accederíamos con lápiz y papel. Podría preguntarse en qué medida la visualización que se ofrece a partir de situaciones basadas en el programa, es ventajosa para el alumno.

La resolución de situaciones problemáticas nuevas y parcialmente nuevas requiere de significados. En general, se han reportado una serie de dificultades relacionadas con la fragilidad de la organización del conocimiento de los individuos (Escudero, 2001, Escudero y Moreira, 2002; Escudero y Jaime, 2003; González y Escudero, 2007a), con la desvalorización de las condiciones de contorno (Escudero y Jaime, 2002, Escudero 2009), con el cambio de modelo conceptual implicado (Escudero y Jaime, 2003), con la diversidad de procesos desarrollados al resolver problemas (Escudero, González y Jaime, 2005), con la interpretación en términos de conocimientos-en-acción al poner en juego determinadas representaciones mentales (Escudero, 2005; Escudero, Moreira y Caballero, 2003 y 2006; González y Escudero, 2007b, etc.).

Resultados empíricos sugieren la posibilidad de movilidad de conocimientos-en-acción generada por propuestas didácticas con fuertes raíces culturales (Escudero y Jaime, 2008), de ahondar desde la génesis del símbolo hasta los mecanismos de la comprensión de una situación física (González y Escudero, 2003), de elementos a favor de la autonomía, como actitud que puede desarrollarse mediante la práctica de actividades de metacognición, además de las habituales de cognición (González y Escudero, 2007a) [2], de que la capacidad de los estudiantes de generar buenas soluciones se ha debido a la puesta en escena de ciertas representaciones mentales (Escudero, 2005, Escudero y Jaime, 2007a y b) no sólo en la universidad. El actual desafío es lograrlo incorporando las nuevas tecnologías.

La integración de saberes propios de un campo disciplinar -Física, Matemática, etc.con el lenguaje informático, nos lleva en cierto sentido "hacia atrás" en relación a la pericia alcanzada por los docentes en ciencias desde un punto de vista más tradicional.

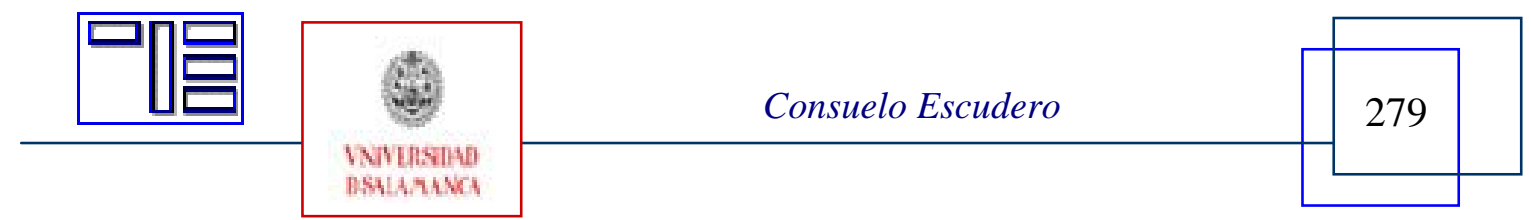


Lo cual nos instala en una zona de inseguridad, de incertidumbre, que puede actuar tanto en forma reactiva como paralizante. Pero la actitud con que los estudiantes asumen este universo de información y comunicación, es un catalizador positivo en tanto fortalece el aprendizaje cooperativo, que es uno de los sustentos de esta renovada manera de ver las cosas.

Por ejemplo, sabemos que el hipertexto permite recorrer caminos no lineales, que aquí la secuencia se construye en función de criterios anclados en modelos mentales. Surge la pregunta ¿son esos caminos la expresión visible de la organización de los conocimientos en el cerebro?. Se trata de un interrogante muy audaz, pero provocativo.

Si consideramos nuevamente que nos encontramos en el umbral de un nuevo campo intelectual, no hay razón que justifique un desapego de todos aquellos conocimientos construidos durante muchos años en áreas tradicionales como son la Matemática y la Física, puesto que son esos constructos los que constituyen el sistema de referencia actual. Es más, hasta el momento se acepta que las "herramientas cognitivas" (Vygotsky, 1978) aún interactúan en forma limitada: "El ordenador como herramienta cognitiva puede ayudar al alumno en su aprendizaje: como suplemento a la memoria humana (...), permite recuperar rápidamente la información (...)” (Jonassen, 2000).

El hecho de no haber logrado aun insertar las tareas cognitivas es una limitación que deberíamos superar, al menos desde nivel secundario, para intentar re-tomar el camino de la creatividad.

\section{3.- LA NECESIDAD DE CONTAR CON UNA MEJOR TEORÍA.}

La asimilación del funcionamiento de la mente con el ordenador [3] es una metáfora de mediados del siglo XX que sigue proporcionando aristas para explicar, en parte, el comportamiento, las reacciones y las actitudes del ser humano. Las recurrentes preguntas acerca de las percepciones, del lenguaje y otras, continúan motorizando las investigaciones acerca de la mente y la cognición humanas. Sin embargo, las proyecciones pedagógicas y la integración al mundo de la enseñanza ha resultado bastante compleja dada las múltiples y diversas variables que entran en juego.

Uno de los fines de las instituciones educativas es dotar a los egresados de las concepciones y competencias necesarias que les permitan insertarse satisfactoriamente en la fuerza productiva de la sociedad, pero esas competencias se han modificado drásticamente. Autores como Ramiszouki (1997) señalan que las nuevas competencias serán: el aprendizaje autónomo y la formación just in time mediante habilidades como análisis metacognitivo de la información y la resolución creativa de problemas. Esto plantea interrogantes interesantes en cuanto a la especificidad de cada una de las producciones tecnológicas y su vinculación con áreas específicas de conocimiento.

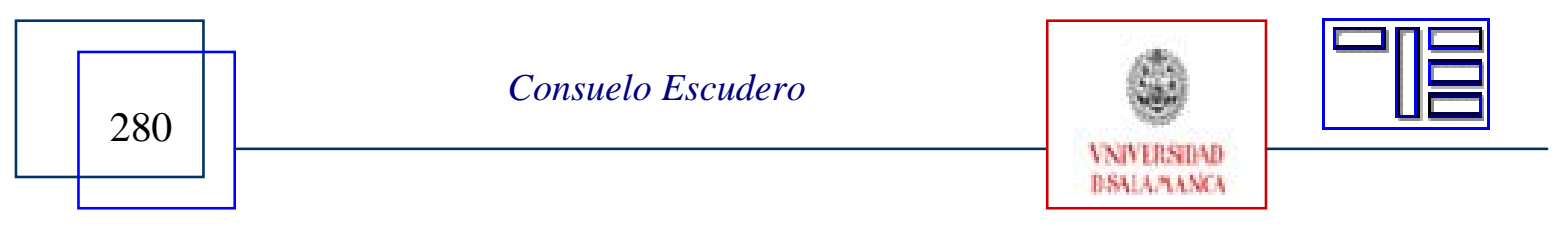


La resolución de problemas con alto contenido semántico en disciplinas como Física no son simples representaciones de objetos o procesos, sino que alojan en sí mismas una compleja trama de conceptos. Es posible intentar aproximaciones hacia su significación desde diferentes puntos de vista:

Por un lado desde los aportes teóricos existentes en relación a las representaciones en virtud de la utilización de nuevas tecnologías de la información y la comunicación.

Las representaciones internas son aquellas que construimos y que usamos las personas frente a una situación, con el objeto de entenderla y además, si es posible de intervenir sobre ella y de comunicar nuestras acciones. Como aludimos a creaciones de la mente también reciben el nombre de representaciones mentales (Escudero, 2005).

En el caso de las representaciones externas se puede hablar de una clasificación muy elemental: representación simbólica (el lenguaje, la numeración) y de representación analógica o pictórica (un dibujo). La selección de un tipo u otro dependerá de varios factores, entre ellos: el objeto a representar, el propósito de la representación y a quien se dirige la comunicación (Greca, 1999).

El mayor dominio de estrategias podría permitir el acceso a actividades de orden superior en colaboraciones posteriores con máquinas inteligentes. El supuesto de esta posibilidad reside en pensar que las capacidades de razonamiento pueden desarrollarse y ser transferidas a otras situaciones distintas, o similares.

Las tecnologías y los entornos culturales que fomentan la atención consciente tienen más posibilidades de producir un residuo cognitivo que aquellas que permiten al individuo la distracción (Salomon, Perkins y Globerson, 1992).

Pea (1985) establece un contraste entre los efectos de una metáfora tecnológica de tipo explicativa y una que reorganiza cognitivamente. En el caso de la computadora (o mejor dicho, de los programas) para que haya una reorganización cognitiva debería haber (o estar) la posibilidad de una asociación en la cual la computadora actúe como herramienta de mediación que permita la realización de nuevas tareas y nuevas operaciones, imposibles de haber sido realizadas sin ella.

Por otro lado, es importante considerar la vertiente psicológico-epistemológica que ubica como cuestión central de la Investigación en Enseñanza de las Ciencias a la formación de conceptos y a la resolución de situaciones problemáticas

Para proceder a la resolución de tareas, de situaciones problemáticas y problemas, los estudiantes deben construir representaciones que propicien explicaciones y predicciones. La construcción de estas representaciones, en tanto, no es una tarea trivial, en gran parte por fundarse en ciertos conocimientos básicos. La idea de que las personas construyen modelos mentales del mundo, relacionando el lenguaje y la percepción con ese mundo a través de la mente, es atrayente.

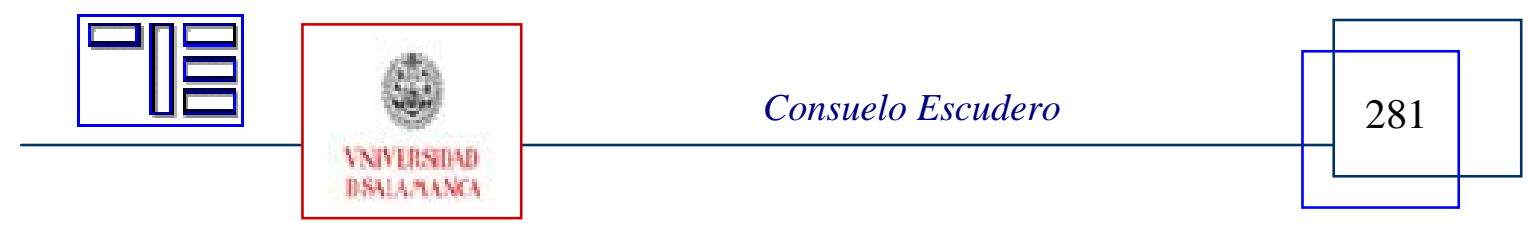


Así los modelos que tienen los alumnos acerca de un concepto, de un algoritmo, de una descripción, por lo general son imprecisos, incompletos e inconsistentes. Precisamente, porque son "modelos provisionales" que están en evolución y que ante una misma instrucción (o no) pueden evolucionar en mayor o menor medida hacia el modelo conceptual científico, que es objeto de la Enseñanza en Ciencias (en nuestro caso).

Entre los individuos lo que se desarrolla son formas de organización de la actividad. El problema de la enseñanza suele ser en gran parte el de llevar al aprendiz a desarrollar sus competencias. Como el concepto de competencia no es suficiente en sí mismo, por una parte se hizo necesario utilizar la noción de actividad y, por otra, se precisaron conceptos para analizar esa actividad. Para desarrollar dicha noción Gèrard Vergnaud ha utilizado el concepto de esquema de Piaget. No es un concepto simple, el mismo término ha sido empleado con variadas significaciones. Para Vergnaud (1996a), Piaget ha aportado al análisis de la "acción en situación” el concepto más decisivo, aunque en su obra no le haya dado todo el alcance que en la actualidad parece oportuno darle [4].

Los textos de Piaget contienen, en general, caracterizaciones sobre lo que es un esquema en acción. Sin embargo, es en los escritos de sus colaboradores en los que se encuentran definiciones y profundizaciones.

\begin{abstract}
La conceptualización de los esquemas no presupone un sujeto que actúa sobre los objetos, sino un organismo que interactúa (dialécticamente [5]) con algo externo a sí mismo. Las acciones repetidas, a través de múltiples encuentros con "realidades" externas al organismo, no sólo generan los esquemas como totalidades organizadas, sino que son, al mismo tiempo organizantes en tanto ese "algo" exterior adquiere significación. (...) Los esquemas de acción se presentan como el nexo que conjunta la triple raíz de su capacidad como órgano asimilador: la raíz biológica, puramente orgánica; la raíz que podríamos llamar "orgánico-psicológica” (las coordinaciones de las acciones), y la raíz empírica (el “mundo” en el cual se ejercen las acciones) García (2000).
\end{abstract}

Mayoritariamente cuando se alude al término esquema [6] se lo hace desde la vertiente computacional. Sin embargo, también concierne a un constructo proveniente de la corriente psicogenética como vemos. El constructo teórico esquema da cuenta de un concepto prototípico en la Teoría de los Esquemas de Rumelhart, en tanto, esquema de acción es retomado y precisado en la Teoría de los Campos Conceptuales de Vergnaud. Uno y otro buscan explicar el razonamiento humano.

En la primera acepción, la dinámica cognitiva se traduciría en formación de nuevos schematas en base a los ya existentes y por reestructuración -a partir de generación espontánea e inducción- y reduciéndose a procesos asociativos; mientras, en la segunda dicha dinámica ocurriría por enriquecimiento conceptual, resguardándose una unidad de significado más molar e introduciéndose la idea de conocimiento-en-acción (conceptosen-acción, teoremas-en-acción, entre otros). Además de las invariantes operatorias [7] el esquema de acción para Vergnaud contiene otros tres componentes más: a) objetivos y subobjetivos; b) reglas para generar y regular comportamientos; c) posibilidad de inferencias: cada simple actuación en cada situación simple implica una inmensa cantidad de computaciones.

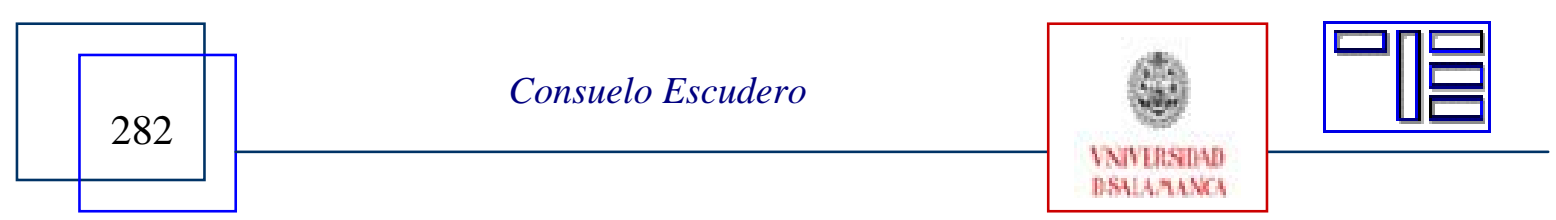


¿Qué es, entonces, un esquema de acción? Un esquema para Vergnaud es una organización invariante de la actuación para una determinada clase de situaciones (1990, 1994, 1996a, 1998) dejando claro que no es la actuación la que es invariante sino la organización de la misma. Esta totalidad dinámica, introducida por Piaget (antes por Kant) no es sólo para formas como ya había propuesto la Gestalt sino que da cuenta tanto de las "habilidades sensorio-motoras" como de las "habilidades intelectuales” y requiere de un riguroso y profundo análisis si se desea comprender las relaciones entre competencias y concepciones.

Vergnaud recupera así la definición de esquema:

- se trata de una totalidad dinámica funcional, es decir, de una unidad identificable de la actividad del sujeto, que se corresponde con una finalidad identificable (1994).

- se trata de un universal, es decir, se reporta a una clase de situaciones, siendo posible, entonces que haya esquemas para todos los dominios de actividad (1998).

El concepto de esquema permite explicar la organización de la actividad del sujeto en una cierta situación encontrándose, entonces, en el centro de la actividad adaptativa de las estructuras cognitivas permitiendo caracterizar mejor los diversos momentos de la psicogénesis en determinado campo conceptual.

La ausencia de una conceptualización adecuada está en el origen de los errores sistemáticos cometidos por los alumnos (Franchi, 1999, Escudero y Moreira, 2002, Escudero y Jaime, 2007a y b). Pero son precisamente las invariantes operatorias [8] las que articulan teoría y práctica; es decir, las que hacen la articulación esencial. Así la percepción, la búsqueda y la selección de información se basarían completamente en el sistema de conceptos-en-acción disponibles en el sujeto (objetos, atributos, relaciones, condiciones, circunstancias) y de teoremas-en-acción subyacentes a su actuación.

No es suficiente apropiarse de un modelo para provocar cambios. Es imprescindible que se lo someta a la prueba empírica, con los recaudos que sea posible prever. Es en la conjunción de ambas perspectivas donde surgen las preguntas que nos invitan a seguir investigando cómo es que se produce el aprendizaje.

Cuando se tiene plena conciencia de la importancia de discriminar claramente cuáles son los contenidos de un modelo, se necesita precisar con mayor profundidad a qué llamamos concepto.

En este sentido, la teoría de los campos conceptuales de Vergnaud hace un aporte que multiplica las posibilidades de conocer la génesis y la comprensión de los conceptos.

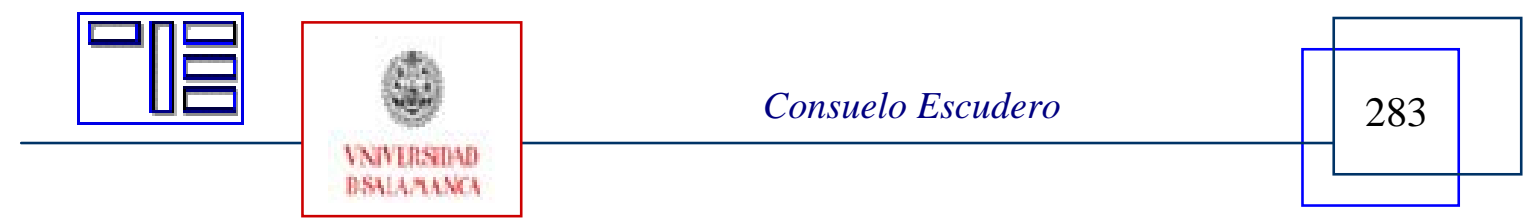


Considera que un concepto es un triplete de tres conjuntos (Vergnaud 1983, 1990; Franchi 1999): $C=(S, I, L)$ donde:

$S$ : conjunto de situaciones que le dan sentido al concepto (el referente);

I: conjunto de invariantes operatorios asociados al concepto (el significado);

$L$ : conjunto de representaciones lingüísticas y no lingüísticas que permiten representar simbólicamente el concepto, sus propiedades, las situaciones a las que él se aplica y los procedimientos que de él se nutren (el significante).

Las fuentes originales de Vergnaud son los estudios de Piaget, fundamentalmente con la noción de esquema como decíamos, y los trabajos de Vygotsky.

Es por esto que se plantea la necesidad de integrar en el programa de trabajo las situaciones conducentes a fortalecer la conceptualización científica y la modelización (entre otras capacidades) con un diseño de gestión del aula que contemple la perspectiva socio cultural, cuyo trazado inicial fue elaborado por Vygotsky.

Actualmente, los fundamentos acuñados por Vygotsky son un referente ineludible en las investigaciones realizadas acerca de la interacción hombre-computadora en entornos de aprendizaje. Las nuevas tecnologías de la comunicación y de la información (NTICs) han renovado la discusión acerca del concepto de aprendizaje. Aparecen preguntas que cuestionan algunos supuestos básicos de la psicología tradicional.

Lo que tradicionalmente ha ocurrido es que el aprendizaje solo ha sido pensado desde el campo de la informática educativa, o de la tecnología educativa, en una relación casi < diádica > con la propia tecnología, como una especie de efecto de la misma. El programa informático sería el entorno y el aprendizaje la consecuencia.

(...) las diferentes aproximaciones a la tecnología se convierten en grandes metáforas que nos indican cómo pensar esas relaciones entre las personas y las máquinas.

(...) se defienden visiones: antropológica, instrumental discursiva, etc. (...) Es evidente que las relaciones entre la tecnología informática y el aprendizaje han estado dominadas por una metáfora instrumental, y, en gran medida, ese ha sido uno de los problemas en la manera de pensar los aprendizajes (Rodríguez Illera, 2005).

Desde el procesamiento de la información Mayer (citado en Rodríguez Illera, 2005) propone algunas orientaciones de sólidos fundamentos teóricos y empíricos. Aún cuando existan aspectos cuestionables, los criterios enunciados para los tipos de enseñanza tienen un correlato bien visible en la práctica docente: aprendizaje multimedia, aprender haciendo y aprendizaje colaborativo.

La configuración de la teoría de los campos conceptuales de Vergnaud tanto como marco teórico alternativo para la investigación en resolución de problemas en Ciencias (Escudero y Moreira, 2004; Escudero, 2005 [9]) como, referente plausible de integrar los modelos mentales de Johnson-Laird $(1983,1990)$ con los esquemas de acción de Vergnaud (Moreira, 2002 y Greca y Moreira, 2002) son los que permiten profundizar en el análisis de la construcción de los conocimientos. Mientras que la perspectiva cognitiva

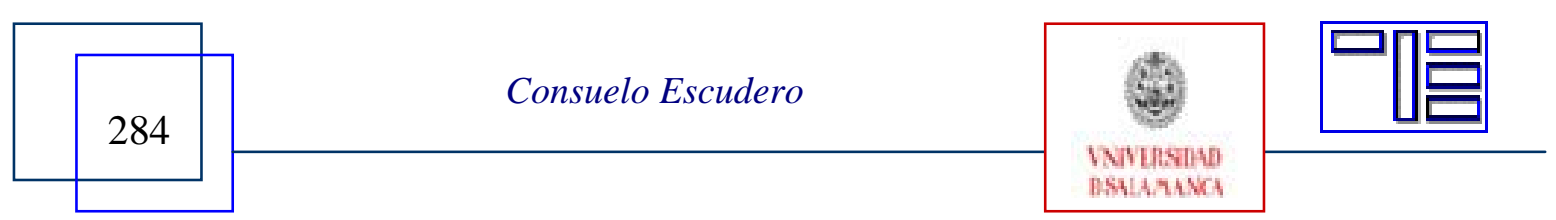


de los instrumentos contemporáneos de Rabardel (2002) brinda algunos constructos para comprender la bidimensionalidad asociada de artefactos y esquemas de utilización.

Sin dejar de tener presente que, el paso desde el asociacionismo al estructuralismo supone correrse de investigar la identificación de conceptos a ocuparse también de su adquisición o formación. Análogamente, podemos proponer que cuando se utilizan nuevas tecnologías supondría pasar de investigar la identificación de entidades mixtas como son los instrumentos -relacionadas a la vez con el individuo y con el artefacto- a ocuparse también de su adquisición o formación; es decir, de la génesis instrumental en términos de Rabardel.

\section{4.- A MODO DE CIERRE.}

En la psicología cognitiva clásica destacan dos grandes vertientes teóricas con variadas contribuciones: la psicología de base computacional y la psicología cognitiva evolutiva o estructural. Las dos han evolucionado en su corta vida siguiendo líneas de desarrollo paralelas, primero sintácticas luego semánticas (Escudero, 2005), con nociones poderosas tales como memoria, cogniciones distribuidas, residuo cognitivo las unas; con enfoques como el desarrollo de los instrumentos contemporáneos y con conceptos de gran claridad como acción, actividad, génesis instrumental; las otras. Ambas siguen guardando una cierta distancia, donde aún no se producen importantes convergencias.

En la psicología contemporánea, es central la relación cognición-acción. Recuperamos el énfasis puesto en la necesidad de comprender las vinculaciones entre los usos de las tecnologías y los efectos cognitivos -entre ellos, el remanente cognitivo- en el marco de la cultura. El surgimiento de la perspectiva del desarrollo de los instrumentos contemporáneos de Rabardel permitiría mostrar los límites de validez de una psicología de la actividad del sujeto, de la persona y sus capacidades. Lo que nos lleva a intentar clarificar más sobre nosotros mismos e iniciaría, a nuestro entender, un nuevo programa de investigación sobre las interacciones hombre-computadora.

En esa compleja relación entre residuo cognitivo, la influencia de las TIC's y la enseñanza que se propone se alcanzan a avizorar algunas cuestiones que podrían iluminar discusiones didácticas, tales como la incorporación de la novedad a través de la problematización y la construcción de criterios propios surgidos en la actividad grupal. La problematización funciona como dinamizador de nuevas preguntas que invitan a buscar caminos alternativos, a establecer nuevas relaciones, a inaugurar criterios para seleccionar la información y la lógica misma.

Nuestros resultados apoyan la toma de conciencia sobre la importancia de re-establecer el papel de la resolución de problemas en la era de las nuevas tecnologías, de la conceptualización de la Física, del modelado y la conceptualización de la Matemática en el aprendizaje de la Física, entre otros aspectos. Decíamos:

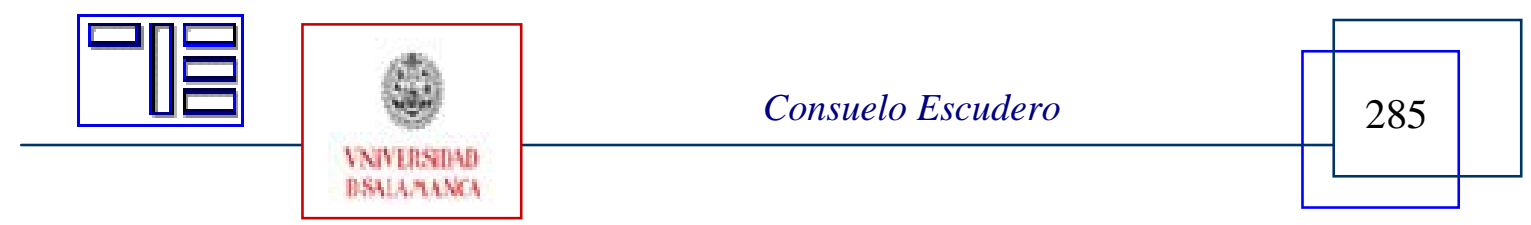


Una posible salida sería la creación de escenarios en los que usar el conocimiento científico sea relevante. Al decir esto estamos incorporando a la matriz del aprendizaje de las ciencias, la mediación social y cultural. Esta perspectiva debería ser considerada de mayor interés a la hora de trabajar.

Muchas veces la invisibilidad de las dificultades en el aprendizaje de conceptos, símbolos, relaciones, imposibilita que se promueva la renovación del quehacer didáctico -e incluso investigativo- mismo (Escudero, 2007).

Pareciera que los interrogantes abiertos nos permiten homologar [10] una problemática que no se circunscribe a la universidad ni a la resolución creativa de problemas, sino que resulta genuina a la hora de pensar propuestas didácticas y sus derivaciones cognitivas cuando se introducen tecnologías, especialmente software, en el aula.

Tradicionalmente se piensa a la tecnología como una prolongación del cuerpo. Actualmente tenemos la opción de sugerirla como prótesis cuasi-mental. Aún cuando los riesgos de producir un letargo intelectual en un mundo dispuesto a esforzarse cada vez menos, vale la pena continuar explorando en qué medida conviene esta alianza mentemáquina para continuar mejorando las posibilidades de aprender.

\section{5.- REFERENCIAS.}

ARAUJO, I.; VEIT, E. y MOREIRA, M. A. (2007): Simulações computacionais na aprendizagem da Lei de gauss para electricidade e da Lei de Ampère em nível de Física General. Revista Electrónica de Enseñanza de las Ciencias, 6 (3), 601629.

BAINBRIDGE, L. (1982): Ironies of automation, proceedings of the IFAC/IFORS/IEA/IFS conference on análisis, design and evaluation of manmachine systems, Baden Baden.

BALLESTEROS, S. (2003): Resolución de problemas y motivación en espacios virtuales. Propuesta de una línea de investigación. Teoría de la Educación: Educación $y$ Cultura en la Sociedad de la Información. http://www3.usal.es/ teoriaeducacion/rev_impresa.htm

BATES, T. (2001): Cómo gestionar el cambio tecnológico. Barcelona, Gedisa.

BUZZO, R. (2007): Estrategia EE (Excel-Euler) en la enseñanza de la Física. Lat. Am. y Phys. Educ., 1(1), 19-23.

CATALDI, Z. y CABERO, J. (2007): Las competencias profesionales en ambientes informáticos para trabajos colaborativos y resolución de problemas. Teoría de la Educación: Educación y Cultura en la Sociedad de la Informción. 8(1), http://www3.usal.es/ teoriaeducacion/rev_impresa.htm
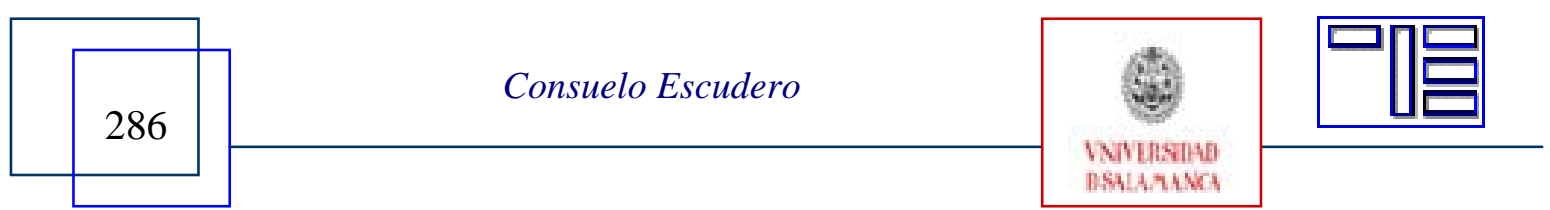
CLOT, Y. (1992): La vigilance peut-elle être automatisée. Performances Humaines et Techniques, septembre, 56-59.

ESCUDERO, C. (2001): Representaciones, modelos mentales y su relación con omisiones, aciertos y errores de actuación al resolver un problema de Física. Enseñanza de las Ciencias, Número Extra, VI Congreso Internacional sobre Investigación en la Didáctica de las Ciencias, Barcelona (España).

ESCUDERO, C. (2005): Inferencias y modelos mentales: un estudio de resolución de problemas acerca de los $1^{\circ}$ contenidos de Física abordados en el aula por estudiantes de nivel medio. Tesis doctoral. Universidad de Burgos (España).

ESCUDERO, C. (2007): Interacción y actividad instrumental vygotskyana en una perspectiva ausubeliana de aprendizaje. Revista de Enseñanza de la Física (Argentina), 20 (1 y 2), 41-54.

ESCUDERO, C. (2009): Las leyes de conservación y el mundo físico: un estudio de caso desde la teoría de los campos conceptuales. VIII Congreso Internacional sobre Investigación en la Didáctica de las Ciencias, Barcelona (España). [Enviado]

ESCUDERO, C.; GONZÁLEZ, S. y JAIME, E. (2005): El análisis de conceptos básicos de Física en la resolución de problemas como fuenet generadora de nuevas perspectivas. Un estudio en dinámica del movimiento circular. Revista Educación y Pedagogía (Colombia), XVII (43), 61-78.

ESCUDERO, C. y JAIME, E. (2002): La comprensión de la situación física en la resolución de un problema integrativo. Memorias VI SIEF, Corrientes (Argentina).

ESCUDERO, C. y JAIME, E. (2003): Elementos para una conceptualización de la noción de cuerpo rígido en la resolución de un problema integrativo. Memorias XIII Reunión Nacional de Educación en Física (REF XIII), Río Cuarto (Córdoba, Argentina). ISBN. 987-1003-15-3.

ESCUDERO, C. y JAIME, E. (2007a): La comprensión de la situación física en la resolución de problemas: Un estudio en dinámica de las rotaciones. Revista Electrónica de Enseñanza de las Ciencias, 6 (1), 1-19. http://www.saum.vigo.es/reec

ESCUDERO, C. y JAIME, E. (2007b): Sonority as variation: study about the conceptualization of physical notions in university students. Journal of Physics Conferences Series (JPCS), 90 012021. IOP Publishing. http:/www.iop.org/EJ/article/1742-6596/90/1/012021/

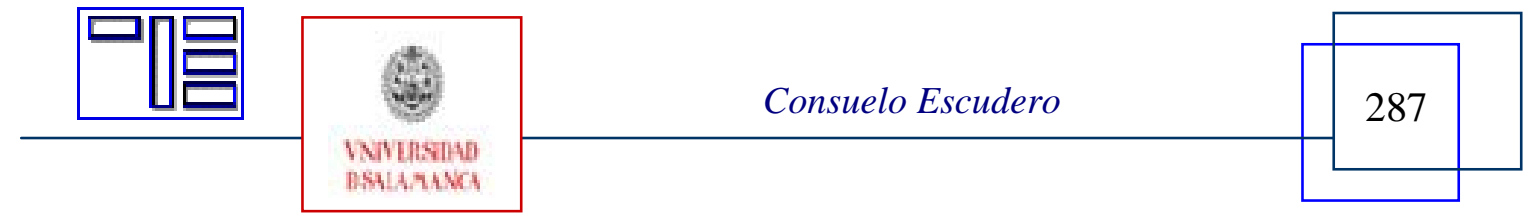


ESCUDERO, C. y JAIME, E. (2008): Conocimientos-en-acción: un estudio en profundidad acerca de la integración de las fuerzas y la energía en cuerpo rígido. IENCI [Enviado para publicación]

ESCUDERO, C. y MOREIRA, M. A. (2002): Resolución de problemas de cinemática en nivel medio: estudio de algunas representaciones. Revista Brasileira de Pesquisa em Educação em Ciências (ABRAPEC), 2 (3), 5-25. http://www.fc.unesp.br/abrapec/revistas

ESCUDERO, C, y MOREIRA, M. A. (2004): La investigación en resolución de problemas: una visión contemporánea. Texto de Apoio $\mathrm{N}^{\circ} 23$ da Universidade de BURGOS/UFRGS, vol. 6, 41-90. En MOREIRA, M. A y CABALLERO, C. (Eds): Actas del PIDEC. Porto Alegre, UFRGS.

ESCUDERO, C; MOREIRA, M. A. y CABALLERO, C. (2003): Teoremas y conceptos-en-acción en clases de Física introductoria en secundaria. Revista Electrónica de Enseñanza de las Ciencias, 2 (3). http://www.saum.vigo.es/reec

ESCUDERO, C; MOREIRA, M. A. y CABALLERO, C. (2009): A research on undergraduate students' conceptualizations of physics notions related to non-sliding rotacional motion. Lat. Am. J. Phys. Educ., Vol 3, $N^{o} 1,1-8$.

FRANCHI, A. (1999): Considerações sobre a teoria dos campos conceituais. En Alcântara Machado, S. D. et al. Educação Matemática: uma introdução. São Paulo, EDUC, 155-195.

GARCÍA, R. (2000): El conocimiento en construcción. Barcelona, Gedisa.

GONZÁLEZ, S. y ESCUDERO, C. (2003): Un estudio sobre el discurso del profesor de Física en clases de resolución de problemas. II Encontro Internacional Lenguaje, Cultura y Cognición: reflexiones para la enseñanza. UFMG, Belo Horizonte (Brasil).

GONZÁLEZ, S. y ESCUDERO, C. (2007a): En busca de la autonomía a través de las actividades de cognición y de metacognición en ciencias. Revista electrónica de Enseñanza de las Ciencias, 6 (2), pp. 310-330. http://www.saum.vigo.es/reec

GONZÁLEZ, S. y ESCUDERO, C. (2007b): Las unidades como parte de un campo conceptual. Primer Encuentro Nacional sobre Enseñanza de la Matemática (1 ENEM) Tandil (Bs. As.), 11 al 13 de abril. Memorias en CD.

GRECA, I. (1999): "Representaciones mentales". Texto de apoyo Nº7. Programa Internacional de Doctorado en Enseñanza de las Ciencias: Universidad de Burgos (España)- UFRGS (Brasil).

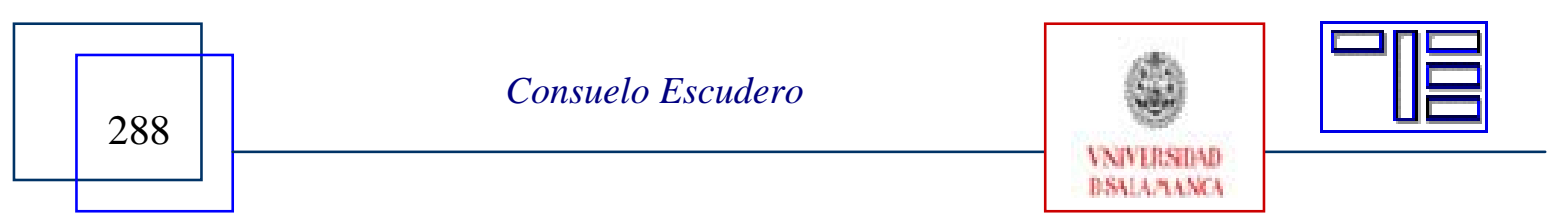


GRECA, I. y MOREIRA, M. A. (2002): Além da deteç̧ão de modelos mentais dos estudantes. Uma proposta representacional integradora. Investigações em Ensino $\begin{array}{llllll}\text { de } & \text { Ciências. } & \text { Brasil, } & \text { Vol. } & 7 & \text { (1). }\end{array}$ http://www.if.ufrgs.br/public/ensino/revista.htm

JIMOYANNIS, A. y KOMIS, V. (2001): Computer simulations in physics teaching and learning: a case study on students'understanding of trayectory motion. Computer and Education 36, 183-204.

JOHNSON-LAIRD, P (1983): Mental Models. Cambridge, Cambridge University Press.

JOHNSON-LAIRD, P. (1988): The Computer and the Mind: An Introduction to Cognitive Science. Collins Sons and Co, Glasgow [Trad Cast: El ordenador y la mente. Introducción a la ciencia cognitiva. Barcelona, Paidós, 1990].

JONASSEN, D. H. (2000): El diseño de entornos constructivistas de aprendizaje. En REIGELUTH, C.(ed) Diseño de la instrucción. Teorías y modelos. Madrid, Aula XXI. Santillana, 225-249.

LION, C. (2006): Imaginar con tecnologías. Buenos Aires, La Crujía Ediciones.

LÓPEZ, M; ESPINOZA, A. y FLORES, K. (2006): Percepción sobre las tecnologías de la información y la comunicación en los docentes de una universidad mexicana del centro universitario del sur de la Universidad de Guadalajara. Revista Electrónica de Investigación Educativa, 8(1), 1-14.

MOREIRA, M. A. (2002): A teoría dos campos conceituais de Vergnaud. Investigações em Ensino de Ciências. Brasil, Vol. 7 (1). Site: http://www.if.ufrgs.br/public/ensino/revista.htm

PEA, R. (1985): Beyons amplification: Using computer to reorganize mental functioning. En : Educational Psychologist, No 20, Norwood.

PEA, R. (2001): Prácticas de la inteligencia distribuida y diseños para la educación. En Salomon, G (comp.): Cogniciones distribuidas. Buenos Aires, Amorrortu.

PERALES, F. y CAÑAL, P. (2000): Didáctica de las Ciencias Experimentales. Editorial Marfil, España.

POZO, J. I. y GÓMEZ CRESPO, M. A. (1998): Aprender y enseñar ciencia. Madrid, Morata.

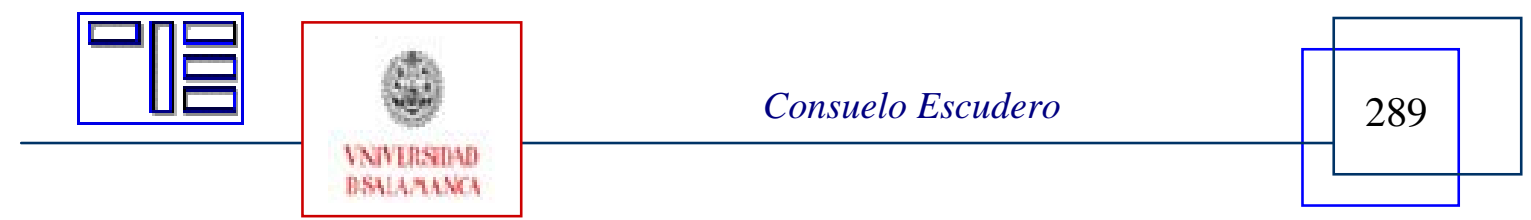


RABARDEL, P. (2002): Los hombres y las tecnologías. Perspectiva cognitiva de los instrumentos contemporáneos. Biblioteca Virtual BV-EEE.

RAMISZOUKI, A. (1997): Web-based distance learning and teaching: Revolutionary invention or reaction to necessity? En KHAN, B. (Ed.): Web Based Instruction (24-40)- Englewood Cliffs, CA: Educational Technology Publications.

RODRÍGUEZ ILLERA, J. L. (2005): El aprendizaje virtual: Enseñar y aprender en la era digital. Rosario-Argentina. Ediciones Homo Sapiens.

SALOMÓN, G. PERKINS, D. y GLOBERSON, T. (1992): Co-participando en el conocimiento: la ampliación de la inteligencia humana con las tecnologías inteligentes, en: Comunicación, Lenguaje y Educación, 13.

SAMAJA, J. (1999): Epistemología y metodología. Buenos Aires: EUDEBA. Edición revisada.

SANTOS, G.; OTERO, M. R. y FANARO, M. A. (2000): ¿Cómo usar software de simulación en clases de Física? Caderno Catarinense de Ensino de Física, 17(1), 50-66.

SIERRA, J. L. (2000): Informática y enseñanza de las ciencias. En PERALES PALACIOS, F. y CAÑAL, P. (eds.): Didáctica de las Ciencias Experimentales. Editorial Marfil, España.

SIERRA, J. L. (2004): Estudio de la influencia de un entorno de simulación por ordenador en el aprendizaje por investigación de la Física en Bachillerato. Tesis doctoral.

http://www.mec.es/cide/espanol/publicaciones/colecciones/investigacion/col167 /col167.pdf

TELLO, J. y PÉREZ, C. (2007): Integración del software modellus a la metodología de modelamineto mental para el aprendizaje de Física. Revista Científica de Educación Chilena, 6 (1), 43-53.

VERGNAUD, G. (1983): Quelques problèmes theóriques de la didactique a propos d'un example: les structures additives. Atelier International d'Eté: Récherche en Didactique de la Physique. La Londe les Maures, França, 26 de junho a 13 de julho.

VERGNAUD, G. (1990) La théorie des champs conceptuels. Recherches en Didactique des Mathématiques, 10 (23), 133-170.

VERGNAUD, G. (1994): Multiplicative conceptual field: what and why? En GUER-

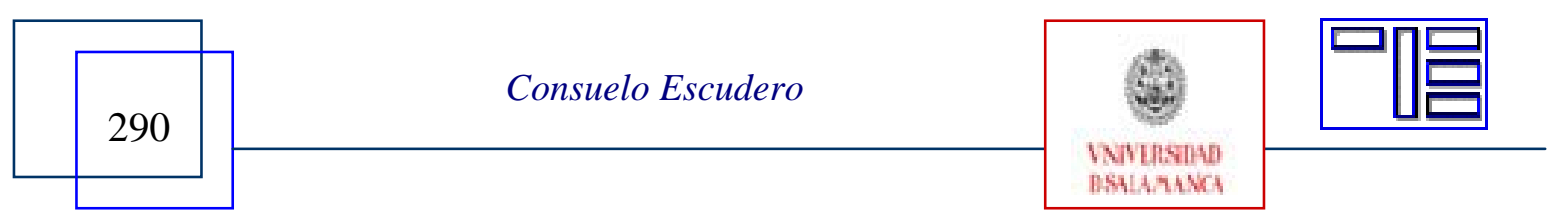


SHON, H. y CONFREY, J. (eds.): The development of multiplicative reasoning in the learning of mathematics (41-59). Albany, NY: State University of New York Press.

VERGNAUD, G. (1996a): Algunas ideas fundamentales de Piaget en torno a la didáctica. Perspectivas, 26(10), 195-207.

VERGNAUD, G. (1996b): A trama dos campos conceptuais na construção dos conhecimentos. Revista de GEMPA, Porto Alegre, N 4, 9-19.

VERGNAUD, G. (1997): Aportes de la psicología del aprendizaje a la tarea docente Entrevista ( $1^{\circ}$ y $2^{\circ}$ parte). Novedades Educativas, 76, 39-40 y n ${ }^{\circ} 77,62-64$. Buenos Aires, Editorial Propietaria Beatriz Kaplan.

VERGNAUD, G. (1998): A comprehensive theory of representation for mathematics education. Journal of Mathematical Behavior, 17(2), 167-181.

VYGOTSKY, L. (1978): Pensamiento y Lenguaje. Buenos Aires, La Pléyade.

VYGOTSKY, L. (1979): El desarrollo de los procesos psicológicos superiores. Barcelona, Crítica.

\section{Notas:}

[1] En la acepción de sobrante, sedimento, vestigio, huella o exceso; y no de restante, desecho, escombro, escoria, saldo o desperdicio. Una analogía estructural puede ser de gran utilidad: La remanencia en ciencias físicas alude a una propiedad de las sustancias ferromagnéticas de conservar cierta imantación una vez que ha cesado la acción inductora de un campo magnético.

[2] Para ello es importante apuntalar la autorregulación de los aprendizajes, lo que favorecería la comprensión de los conceptos involucrados en la resolución de problemas. El estudio se ha llevado a cabo en una temática de Química General: estequiometría.

[3] Quizás sea conveniente recordar aquí que el ordenador cognitivo es, en última instancia, un dispositivo para convertir energía en símbolos, símbolos en otros símbolos y símbolos en acciones.

[4] Notar que se dice Piaget y no Vygotsky. En Vygotsky y seguidores el concepto de actividad refiere esencialmente al trabajo, a la actividad colectiva del trabajo. Es decir, no se encuentra el equivalente a los conceptos de esquema e invariante operatoria con la precisión con que fueron planteados por Piaget. “A pesar del hecho de que uno siempre presenta a Vygotky como el ‘teórico de la actividad'” (Vergnaud, 1997).

[5] Agregado nuestro.

[6] Vocablo traducido del inglés schematas y del francés schémas, a diferencia del de shème que traducimos como esquema de acción que tiene, por cierto, otro origen. Esta distinción no es trivial, con frecuencia suelen equipararse. Una discusión más detallada sobre estas diferencias puede encontrarse en Escudero (2005), Escudero y Moreira (2004).

[7] Invariantes operatorias o conocimientos-en-acción -como también son conocidos- dirigen el reconocimiento de los elementos pertinentes de la situación y la toma de información sobre la situación a tratar. Son los conocimientos contenidos en los esquemas (Vergnaud, 1990; 1994 y 1996b).

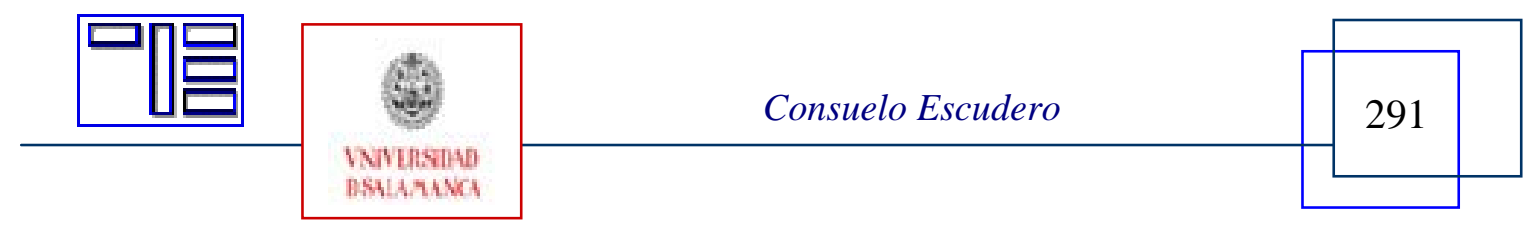


Revista Electrónica Teoría de la Educación.

Educación y Cultura en la Sociedad de la Información.

http://www.usal.es/teoriaeducacion

Vol. 10. No 1. Marzo 2009

[8] Las invariantes operatorias se refieren a objetos, propiedades y relaciones que se mantienen a través de una serie de variaciones (o situaciones). Estos conocimientos, obviamente, no aparecen al modo de su función disciplinar -física, matemática, química, etc. -, sino que son utilizados en la acción y en la resolución de tareas, situaciones, problemas. Un teorema-en-acción es una proposición considerada como verdadera sobre lo real; un concepto-en-acción es una categoría de pensamiento considerada como pertinente (Vergnaud 1996a, 1998).

[9] En 2004 elaboramos un cuadro teórico en el marco de una tesis de doctorado (Escudero, 2005) que dio lugar a un capítulo de libro (Escudero y Moreira, 2004) que posibilita el ensamble y entramado de contribuciones teóricas concernientes a la psicología cognitiva, la psicología del desarrollo, la resolución de problemas, la representación lingüística y simbólica de los conceptos y su relación con la didáctica.

[10] Notar que no se dice inducir. Luego, se sostiene que "el proceso de inferir a partir de 'muestras' es un proceso analógico y no inductivo, queriendo significar que el presupuesto de base contiene un juicio de analogía que se expresa así: ‘esta muestra es representativa'. Ahora bien, para poder afirmar que una muestra es representativa debemos tener un conocimiento mínimo suficiente acerca del tipo de variabilidad del atributo estudiado en el universo.” (Samara, 1999). Pueden consultarse más precisiones en la obra referenciada.

Para citar el presente artículo puede utilizar la siguiente referencia:

ESCUDERO, Consuelo (2009). Una mirada alternativa acerca del residuo cognitivo cuando se introducen nuevas tecnologías. El caso de la resolución de problemas en ciencias. En SAN MARTÍN ALONSO, A. (Coord.) Convergencia Tecnológica: la producción de pedagogía high tech [monográfico en línea]. Revista Electrónica Teoría de la Educación: Educación y Cultura en la Sociedad de la Información. Vol. 10, nº 1. Universidad de Salamanca [Fecha de consulta: $\mathrm{dd} / \mathrm{mm} / \mathrm{aaaa}$. http://www.usal.es/ teoriaeducacion/rev_numero_10_01/n10_01_escudero.pdf ISSN: 1138-9737

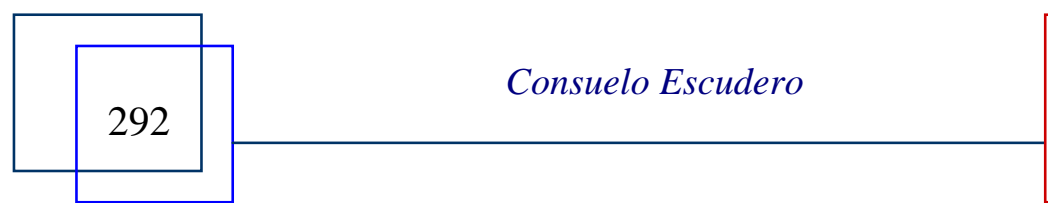

